\title{
International Journal of Engineering Technologies and Management Research
} IJETMR

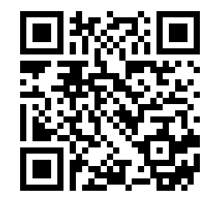

NANO ROBOTS IN MEDICINE: A REVIEW

Ved Prakash Upadhyay ${ }^{1}$, Mayank Sonawat ${ }^{1}$, Kalpana V.Singh ${ }^{2}$, Ramchander Merugu ${ }^{* 3}$

${ }^{1}$ Government Engineering College, Bikaner, Rajasthan, India

${ }^{2}$ Govt. Madhav Science P.G.College Ujjain, M.P., India

${ }^{3}$ Department of Biochemistry, Mahatma Gandhi University, Nalgonda, Telangana, India

Abstract:

In coming years, nanotechnology is likely to have a significant impact in different fields like medicine and electronics. Nanorobotics is emerging as a demanding field dealing with miniscule things at molecular level. Nano robots perform a specific task with precision at nanoscale dimensions. Nano robots are especially used for studies on Alzheimer disease and cancer treatments. These can be seen as the first Nano medicines, with potential application in medicine. Present day treatment includes surgeries which are considered outdated when compared to today's technology.

Keywords: Nanorobots; Design; Types; Applications.

Cite This Article: Ved Prakash Upadhyay, Mayank Sonawat, Kalpana V.Singh, and Ramchander Merugu. (2017). "NANO ROBOTS IN MEDICINE: A REVIEW." International Journal of Engineering Technologies and Management Research, 4(12: SE), 27-37. DOI: 10.29121/ijetmr.v4.i12.2017.588.

\section{Introduction}

The opportunities of Nanotechnology include designing Nano sized, bio responsive systems which can diagnose and then deliver drugs to the site of location. Nanotechnology is an area which is changing vision of medical science. Nanotechnology includes characterization, production and application of nanoparticles in science fields [1]. They possess unique electrical, optical and biological properties. Many chemical and physical methods of synthesis have been reported in the literature [2-8]. Due to the ecofriendly nature of synthesis by biological methods, plant extracts, bacteria, algae, fungi and enzymes have also been exploited [8-23]. Biological methods for synthesizing of silver nanoparticles can be considered to be economical and sustainable alternative to the existing chemical or physical methods. Designs of Nano robots include onboard sensors, power supplies, motors, manipulators and molecular computers. Nano robot is an excellent tool for future medicine. We can envision a day when you could inject billions of these Nano robots that would float around in your body. Nano robots could carry and deliver drugs into defected cells. These nano robots will be able to repair tissues, clean blood vessels and airways, transform our physiological capabilities and even potentially counter act the aging process. Nanotechnology as an emerging tool in medicinal applications especially for diabetes, arteriosclerosis, gene therapy, dentistry and cancer showed how actual developments in new manufacturing technologies are enabling innovative works which may help in constructing 
and employing nano robots most effectively for biomedical problems. Nano robots applied to medicine hold a wealth of promise from eradicating disease to reversing the aging process. Nano robots are also candidates for industrial applications. They will provide personalized treatments with improved efficacy and reduced side effects that are not available today. They will provide combined action drugs marketed with diagnostics, imaging agents acting as drugs, surgery with instant diagnostic feedback. The advent of molecular nanotechnology will again expand enormously the effectiveness, comfort and speed of future medical treatments while at the same time significantly reducing their risk, cost, and invasiveness. This science might sound like a fiction now, but nanorobotics has strong potential to revolutionize healthcare, to treat disease in future. It opens up new ways for vast, abundant research work. Nanotechnology will change health care and human life more profoundly than other developments. Consequently, they will change the shape of the industry, broadening the product development and marketing interactions between Pharma, Biotech, Diagnostic and Healthcare industries. In this review, Nanorobot's mechanism and applications of them in medicine for curing diseases will be discussed.

\section{Composition of Nanorobots}

\subsection{Biochip}

Synthesis involves the joint use of Nano electronics, photolithography, and new biomaterials. It can be used for manufacturing of Nano robots for common medical applications, such as for surgical instrumentation, diagnosis and drug delivery. Electronics industries currently use biochips for manufacturing. Nano robots with biochips can be integrated in Nano electronics devices, which will allow tele-operation and advanced capabilities for medical instrumentation $[8,11]$.

\subsection{Bacteria Based}

This approach uses biological microorganisms, like Escherichia coli bacteria. The model uses a flagellum for propulsion purposes. The use of electromagnetic fields is normally applied to control the motion of this kind of biological integrated device [8-9]

\subsection{Positional Nano Assembly}

Robert Freitas and Ralph Merkle in 2000 are developing a practical research agenda specifically aimed at developing positional-controlled diamond mechanic synthesis and diamonded Nano factory that would be capable of building diamonded medical Nano robots.

\subsection{Nubots}

Nubot is an abbreviation for "nucleic acid robots. "Nubots are synthetic robotics devices at the nanoscale. Representative Nubots include the several DNA walkers reported by Ned Seaman's group at NYU, Niles Pierce's group at Caltech, John Reif's group at Duke University, Chengde Mao's group at Purdue, and Andrew Turberfield's group at the University of Oxford [8, 9] 
1) To cure HIV, Cancer, and other harmful diseases.

2) Nano robots will restore lost tissue at the cellular level.

3) Useful for monitoring, diagnosing and fighting sickness.

4) Able to monitor neuro-electric signals and stimulate bodily systems.

\section{Mechanism of Nanorobots}

The research and development of Nano robots with embedded Nano biosensors and actuators is considered a new possibility to provide new medical devices for doctors. Controls are sought to effectively advance new medical technologies [22, 23]. Development of microelectronics in the 1980s has led to new tools for biomedical instrumentation [24, 25]. Further miniaturization towards integrated medical systems, providing efficient methodologies for pathological prognosis can be designed [26-28]. The use of micro devices in surgery and medical treatments is a reality which has brought many improvements in clinical procedures in recent years [29]. Catheterization has been successfully used as an important methodology for heart and intracranial surgery [30-32]. Now the advent of bimolecular science and new manufacturing techniques is helping us to advance the miniaturization of devices from micro to Nano electronics. Biomedical sensors are being operated by the latest technology which forms the basis for designing bimolecular actuators [33-35]. A first series of nanotechnology prototypes for molecular machines are being investigated in different ways [18, 36-38], along with some devices for propulsion and sensing are also being studied by some workers [18, 36-39, 41]

Nano robot Architecture: The medical Nano robot for biohazard defense should comprise a set of integrated circuit block as an ASIC (application-specific integrated circuit). The architecture has to address functionality for common medical applications [18], providing asynchronous interface for antenna, sensor, and a logic Nano processor, which is able to deliberate actuator and ultrasound communication activation when appropriate. The number of Nano devices to integrate a Nano robot should keep the same hardware sizes with regard to inside body operation applicability [3-6, 35, 47].

\section{Medical Applications of Nanorobots}

Nano robots are expected to enable new treatments for patients suffering from different diseases, and will result in a remarkable advance in the history of medicine. Nanoparticles, liposomes and dendrimers are some nanomaterials being investigated for use in Nano medicine. Nanotechnology has provided the possibility of delivering drugs to specific cells using nanoparticles.

Recent developments in the field of bimolecular computing have demonstrated the feasibility of processing logic tasks by biocomputers. Studies targeted at building biosensors and Nano-kinetic devices required to enable medical nanorobotics operation and locomotion, have also been progressing [38]. In recent years, the potential of nanotechnology has indeed motivated many governments to devote significant resources to this new field. The U.S. National Science Foundation has launched a program in "Scientific Visualization" in part to harness 
supercomputers in picturing the Nano world [3, 53]. In order to build electronics at nanoscales, firms are collaborating to produce new Nano products. Such companies include IBM, PARC, Hewlett Packard, Bell Laboratories, and Intel Corp. The use of Nano robots may advance biomedical intervention with minimally invasive surgeries and help patients who need constant body functions monitoring, or improve treatments efficiency through early diagnosis of possible serious diseases. For example, the Nano robots may be utilized to attach on transmigrating inflammatory cells or white blood cells, thus reaching inflamed tissues faster to assist in their healing process

Nano robots will be applied in chemotherapy to combat cancer through precise chemical dosage administration, and a similar approach could be taken to enable Nano robots to deliver anti-HIV drugs [18-20, 54, 65]. Nano robots could be used to process specific chemical reactions in the human body as ancillary devices for injured organs. Monitoring diabetes and controlling glucose levels for patients will be a possible application of Nano robots. Nano robots might be used to see and break kidney stones. Another important possible feature of medical Nano robots will be the capability to locate atherosclerotic lesions in stenos blood vessels, particularly in the coronary circulation, and treat them either mechanically, chemically or pharmacologically. Cardiovascular problems are generally correlated with the obesity, human sedentary lifestyle, or hereditary characteristics [31, 43, and 61].

\section{Simulation}

The use of micro devices in surgery and medical treatments is a reality which brought many improvements for clinical procedures in the last few years. For example, the catheterization has been used as an important methodology for many cardiology procedures in the same way as the development of micro technology [63] has lead on the 80's to new tools for surgery, now nanotechnology will equally permit further advances providing better diagnosis, and new devices for medicine through the manufacturing of nanoelectronics [8-12]. Nano robots may be considered as the most suitable tool for specialists to solve several problems in medicine in the coming few year here including cardiology interventions and medical analyses. In our study, the Nano robot includes external sensors to inform it of collisions and to identify when it has encountered a chemical signal or abrupt changes of temperature for targeted areas. As a practical approach for medicine, thermal and chemical parameters from the patient's body are used for the Nano robot activation. It is well known that there is significant temperature heterogeneity over inflamed plaque surfaces. They are typically hotter. The temperature difference at the site of the lesion from the core temperature can reach up to $\sim 2^{\circ} \mathrm{C}$. Hence, in order to simulate various levels of inflammation, it was used for different wall temperatures in the atherosclerotic plaque region and the temperature distribution in the stenosed coronary artery was calculated. Significant temperature gradients were found in the recirculation zone, following the stenosis [52-55]. The Trans cardiac concentration gradient of some soluble adhesion molecules has been recently found to be correlative with the progression of coronary atherosclerosis. Therefore, their concentration in the blood vessel is also monitored, using a uniform distribution release from the plaque. In a similar manner, the concentrations of some specific proinflammatory cytokines is monitored, whose elevated concentrations are known as an evidence of formation of atherosclerotic lesions. The Nano robots swim in a near-wall region searching for the atherosclerotic lesion. The atherosclerotic lesion was reduced due to Nano robot's activation. 
The parameters generated from the CFD simulation, namely velocities, temperature, signaling values, pro-inflammatory cytokines and soluble adhesion molecules concentrations, are transferred to the NCD [3,5-7, 36] simulator to be included into the Nano robots operating environment [45-47]. As the Nano robot should perform a pre-defined task in a specific target area, the trigger must be activated when the Nano robot is as close as possible to the target. The Nano robot motion takes the advantage of the blood flow velocity profile in such areas, which shows significantly lower velocities. Thus, the rapid activation could result in lower demand of energy. Optimization of control algorithms and activating triggers is the key for rapid behavior response in minimal energy cost. The optimal trigger values are defined running the Nano robots control programs. Therefore, the investigated stenos artery models provide important information useful to Nano robot manufacturing design in terms of sensors and actuators. The Nano robot's activation goal is to decrease the artery occlusion.

\section{Nanorobotics in Dentistry}

The growing interest in the future of dental applications of nanotechnology is leading to the emergence of a new field called Nano dentistry. Nano robots induce oral analgesia, desensitize tooth; manipulate the tissue to re-align and straighten irregular set of teeth and to improve durability of teeth. Further Nano robots can be used to do preventive, restorative and curative procedures [23-27]. Nano dental techniques involve many tissue engineering procedures for major tooth repair. Mainly nanorobotics manufacture and installation of a biologically autologous whole replacement tooth that includes both mineral and cellular components leading to complete dentition replacement therapy.

\subsection{Tooth Durability and Appearance}

Nano dentistry has given material that is nanostructured composite material, sapphire which increases tooth durability and appearance. Upper enamel layers are replaced by covalently bonded artificial material such as sapphire. This material has 100 to 200 times the hardness and failure strength than ceramic. Like enamel, sapphire is a somewhat susceptible to acid corrosion. Sapphire has best standard whitening sealant, cosmetic alternative. New restorative Nano material to increase tooth durability is Nanocomposites [32, 47, 64-66]. This is manufactured by Nano agglomerated discrete nanoparticles that are homogeneously distributed in resins or coatings to produce nanocomposites. The Nano filler include an alum inosilicate powder having a mean particle size of about $80 \mathrm{~nm}$ and a 1:4 ratio of alumina to silica. The Nano filler has a refractive index of 1.503, it has superior hardness, modulus of elasticity, translucency, esthetic appeal, excellent color density, high polish and 50\% reduction in filling shrinkage. They are superior to conventional composites and blend with a natural tooth structure much better.

\subsection{Nano Impression}

Impression material is available with nanotechnology application. Nano filler are integrated in the vinylpolysiloxanes, producing a unique addition siloxane impression material. The main advantage of material is it has better flow, improved hydrophilic properties hence fewer voids at margin and better model pouring, enhanced detail precision. 


\section{Nano Robots for Brain Aneurysm}

For brain aneurysm prognosis, Nano robots need to track the vessel endothelial injury before a subarachnoid hemorrhage occurs. These changes on chemical concentration are used to guide the Nano robots to identify brain aneurysm in the early stages of development.

\section{Nanorobots in Cancer Detection and Treatment}

Cancer can be successfully treated with current stages of medical technologies and therapy tools. However, a decisive factor to determine the chances for a patient with cancer to survives: how earlier it was diagnosed; what means, if possible, a cancer should be detected at least before the metastasis has begun. Another important aspect to achieve a successful treatment for patients is the development of efficient targeted drug delivery to decrease the side effects from chemotherapy [35, 38, 43-47]. Considering the properties of Nano robots to navigate as blood borne devices, they can help on such extremely important aspects of cancer therapy. Nano robots with embedded chemical biosensors can be used to perform detection of tumors cells in early stages of development inside the patient's body. Integrated Nano sensors can be utilized for such a task in order to find intensity of E-cadherin [62-64] signals. Therefore, a hardware architecture based on Nano bioelectronics is described for the application of Nano robots for cancer therapy. Analyses and conclusions for the proposed model are obtained through real time 3D simulation [22-27].

\section{Nanorobots in Gene Therapy}

Medical Nano robots can readily treat genetic diseases by comparing the molecular structures of both DNA and proteins found in the cell to known or desired reference structures [18-20, 64]. Any irregularities can then be corrected, or desired modifications can be edited in place. In some cases, chromosomal replacement therapy is more efficient than repair. Floating inside the nucleus of a human cell, an assembler-built repair vessel performs some genetic maintenance. Stretching a supercoil of DNA between its lower pair of robot arms, the Nano machine gently pulls the unwound strand through an opening for further analysis. Upper arms, meanwhile, detach regulatory proteins from the chain and place them in an intake port. The molecular structures of both DNA and proteins are compared to information stored in the database of a larger nano computer positioned outside the nucleus and connected to the cell repair ship by a communications link. Irregularities found in either structure are corrected and the proteins reattached to the DNA chain [56-58], which re-coils into its original form. With a diameter of only 50 nanometers, the repair vessel would be smaller than most bacteria and viruses, yet capable of therapies and cures well beyond the reach of present-day physicians. With trillions of these machines coursing through a patient's blood stream, "internal medicine" would take on new significance. Disease would be attacked at the molecular level, and such maladies as cancer, viral infections and arteriosclerosis could be wiped out.

\section{Nanorobots in Surgery}

Surgical Nano robots could be introduced into the body through the vascular system or at the ends of catheters into various vessels and other cavities in the human body [28-30] a surgical 
Nano robot, programmed or guided by a human surgeon, could act as a semi-autonomous on-site surgeon inside the human body. Such a device could perform various functions such as searching for pathology and then diagnosing and correcting lesions by Nano manipulation, coordinated by anon-board computer while maintaining contact with the supervising surgeon via coded ultrasound signals [54-57]. The earliest forms of cellular Nano surgery are already being explored today. For example, a rapidly vibrating $(100 \mathrm{~Hz})$ micropipette with a $<1$ micron tip diameter has been used to completely cut dendrites from single neurons without damaging cell viability. Taxonomy of roundworm neurons was performed by femtosecond laser surgery, after which the axons functionally regenerated. A femtolaser acts like a pair of "Nano-scissors" [6367] by vaporizing tissue locally while leaving adjacent tissue unharmed.

\section{Nanomedicine}

Potential applications for nanorobotics in medicine include early diagnosis and targeted drug delivery for cancer biomedical instrumentation, surgery, pharmacokinetics, monitoring of diabetes, and health care in such plans, future medical nanotechnology is expected to employ Nano robots injected into the patient to perform treatment on a cellular level. Such Nano robots intended for use in medicine should be non-replicating [65-67], as replication would needlessly increase device complexity, reduce reliability and interfere with the medical mission. Instead, medical Nano robots are posited to be manufactured in hypothetical, carefully controlled Nano factories in which Nano scale machines would be solidly integrated into a supposed desktopscale $[61,63]$ machine that would build macroscopic products.

\section{Disadvantages}

1) The initial design cost is very high.

2) The design of the Nano robot is a very complicated one.

3) Hard to Interface, customize and design and is complex.

4) Privacy is the other potential risk involved with Nano robots. As Nano robot's deals with the designing of compact and minute devices, there are chances for more eavesdropping [66-68] than that already exists.

5) Electrical systems can create stray fields which may activate bioelectric-based molecular recognition systems in biology. Electrical Nano robots are susceptible to electrical interference from external sources such as RF or electric fields, EMP pulses [68], and stray fields from other in vivo electrical devices [57-58].

6) Nano robots can cause a brutal risk in the field of terrorism. The terrorism and antigroups can make use of Nano robots as a new form of torturing the communities as nanotechnology also has the capability of destructing the human body at the molecular level.

7) The idea of introducing small submarines through the blood vessels has been captured in many films. But the blood at nanoscale becomes viscous and sticky fluid which does not let the submarine to drive along the vessels. Another phenomenon that would not let the submarine to travel is the Brownian movement of the molecules; the collisions between molecules are incontrollable and unpredictable [1-3]. In the last decade, progress in developing nano sized hybrid therapeutics and drug delivery systems has been used for biomedical applications. These machines are expected to be highly efficient, controllable, 
economical in mass production and fully operational with minimal supervision [5]. These ultra-miniature robotic systems and nano mechanical devices will be the biomolecular electro-mechanical hardware of future.

\section{Conclusion}

Future healthcare will make use of sensitive new diagnostics for an improved personal risk assessment. Highest impact can be expected if those major diseases are addressed first, which impose the highest burden on the aging population: cardiovascular diseases, cancer, musculoskeletal conditions, neurodegenerative and psychiatric diseases, diabetes and viral infections. Nano medicine holds the promise to lead to an earlier diagnosis, better therapy and improved follow up care, making the health care more effective and affordable. Nano medicine will also allow a more personalized treatment for many diseases, exploiting the in-depth understanding of diseases on a molecular level.

\section{References}

[1] Requicha A.A.G., "Nano robots, NEMS and Nano assembly", Proceedings of the IEEE, 91(11):1922-1933, 2003.

[2] Check, E., "US urged to provide smallpox vaccines for emergency crews. News," Nature, 417 (6891): 775-776, 2002.

[3] Earhart, K.C.; Beadle, C.; Miller, L.K.; Press, M.W.; Gary, G.C.; Ledbetter, E.K.; Wallace, M.R. "Outbreak of influenza in highly vaccinated crewof US Navy ship". Emerge. Infect. Dis., 7(3):463-465, 2001.

[4] Hillman, M.R. "Overview: cause and prevention in bio warfare and bioterrorism". Vaccine, 20 (25-26):3055- 3067, 2002.

[5] Cavalcanti, A.; Shirinzadeh, B.; Freitas Jr., R.A.Kratky, L.C. "Medical Nano robot architecture based on Nano bioelectronics". Recent Pat. Nanotechnology Bentham Science, 1(1):1-10, 2007.

[6] Oxford, J.S.; Sefton, A.; Jackson, R.; Innes, W.Daniels, R.S.; Johnson, N.P.A.S. "World War I may have allowed the emergence of "Spanish "influenza." Infect.Dis. 2(2):111-114, 2002.

[7] Ahuja, S.P.; Myers, J.R. A "survey on wireless grid computing". J. Supercomputer. 37 (1), 321,2006

[8] Brindle, J.A. Tactical military communications. IEEE Common. Mag. 30 (1), 62-72, 1992.

[9] Couvreur, P.; Vauthier, C. "Nanotechnology: intelligent design to treat complex disease". Pharm. Res. 23 (7), 1417-1450, 2006

[10] Fienberg, S.E.; Shmueli, G. "Statistical issues and challenges associated with rapid detection of bioterrorist attacks". Stat. Med., 24 (4), 513-529, 2007

[11] Geddes, A.M. "The history of smallpox". Clin. Dermatol. 24 (3), 152-157, 2006

[12] O'Toole, T.; Ingles by, T.V. Epidemic response scenario: decision making in a time of plague. Public Health Rep. 116, 92-103, 2001

[13] Cowell, G.; Ammon, C.E.; Hen Gartner, N.W.Hyman, J.M. "Transmission dynamics of the great influenza pandemic" of 1918 in Geneva Switzerland: assessing the effects of hypothetical interventions. J. Theor. Biol., 241 (2), 193-204, 2006

[14] Kilter, M.E.; Gavinio, P.; Lavanchy, D. "Influenza and the work of the world health organization". Vaccine 20, S5-S14, 2002

[15] Yan, X.; Zou, Y. "Optimal and sub-optimal quarantine and isolation control in SARS epidemics". Math. Compute. Model., 47 (1), 235-245, 2008

[16] Yamamoto, N. "Method and apparatus for detecting cancer, influenza, or HIV based on. Nacetyl-galactosaminidase detection". 5998132USAug 1999 
[17] Webby, R.J.; Webster, R.G. "Are we ready for pandemic influenza?" Science 302 (5650), 15191522,2009

[18] Cavalcanti, A.; Shirinzadeh, B.; Fukuda, T.; Ikeda, S. Hardware architecture for Nano robot application in cerebral aneurysm. IEEE-Nano 2007 Int. Conf.Nanotechnol pp. 237-242, Hong Kong, Aug.2007

[19] Goicoechea, J.; Zamarreño, C.R.; Matias, I.R.; Arregui, F.J. "Minimizing the photo bleaching of self-assembled multilayers for sensor applications." Sens. Actuator B-Chem., 126 (1), 41-47, 2007.

[20] Leary, S.P.; Liu, C.Y.; Apuzzo, M.L.I. Toward the emergence of Nano neurosurgery: Part III. Nano medicine: targeted Nano therapy, Nano surgery, and progress toward the realization of Nano neurosurgery. Neurosurgery, 58 (6), 1009-1025.,2006

[21] Curtis, A.S.G.; Dalby, M.; Gadegaard, N. "Cell signaling arising from Nano topography: implications for nonmedical devices. Nano medicine, 1 (1), 67-72., 2006

[22] Adamson, P.B.; Conti, J.B.; Smith, A.L.; Abraham, W.T.; Aaron, M.F.; Aranda, J.M.; Baker, J.; Bourge, R.C.; Warner-Stevenson, L.; Sparks, B.“ Reducing events in patients with chronic heart failure (Reduce) study design: continuous hemodynamic monitoring with an implantable defibrillator. Clin. Car diol., 30 (11), 567-575.,2007

[23] Ohki, T.; Ouriel, K.; Silveira, P.G.; Katzen, B.; White, R.; Criado, F.; Dietrich, E. "Initial results of wireless pressure sensing for endovascular aneurysm repair: the APEX trial-acute pressure measurement to confirm aneurysm sac exclusion. J. Vasc. Surg. 45(2), 236-242.,2007

[24] Ramcke, T.; Rosner, W.; Risch, L. Circuit configuration having at least one Nano electronics Component and a method for fabricating the component. 6442042US, Aug 2002.

[25] Das, S.; Gates, A.J.; Abdu, H.A.; Rose, G.S.;Picconatto, C.A.; Ellenbogen, J.C. "Designs for ultra-tiny,special-purpose Nano electronic circuits". IEEE Trans. Circuit's Syst. I-Regul. Pap.54 (11), 2528-2540.,2002

[26] Narayan, R.J.; Kumta, P.N.; Sfeir, C.; Lee, D.-H. Olton, D.; Choi, D. Nanostructured ceramics in medical devices: applications and prospects. JOM56 (10), 38-43., 2004

[27] Hede, S.; Huilgol, N. "Nano: the new nemesis of cancer. J. Cancer Res. Ther.2 (4), 186-195.1998

[28] Vaughn, J.R. "Over the horizon: potential impact of emerging trends in information and communication technology on disability policy andpractice." National Council on Disability, WashingtonDC, Dec.25 (1),2006

[29] Murphy, D.; Challacombe, B.; Nedas, T.; Elhage, O.; Althoefer, K.; Seneviratne, L.; Dasgupta, P."Equipment and technology in robotics". Arch.Esp.Urol.,60 (4), 349-354., 2006

[30] Roue, C.C. "Aneurysm liner. 6350270US Feb.200831) McNeil J.S., "Nano robot Pioneer Reveal Status of Simulator, Stem Cell Work," Nano Biotech News, Vol.2, n. 36, pp. 4-5, September 2004, www.nanorobotdesign.com/article/Nano biotech pdf

[31] Cavalcanti A., Freitas Jr. R.A., "NanoroboticsControl Design: A Collective Behavior Approach forMedicine", IEEE Transactions on Nanobioscience, Vol. 4, no. 2, pp. 133-140, June2005.

[32] Casal A., Hogg T., Cavalcanti A., "Nano robots as Cellular Assistants in Inflammatory Responses", inProc. IEEE BCATS Biomedical Computation at Stanford 2003. Symposium, IEEE Computer Society, Stanford CA, USA, Oct. 2003.

[33] Katz E., Ricin A., Heleg-Shabtai V., Willner I., Bückmann A.F., "Glucose Oxidase Electrodes via Reconstitution of the Apo-Enzyme: Tailoring of17Novel Glucose Biosensors", Anal. Chime. Acta.385, 45-58, 1999.

[34] Mc Devitt M.R., Ma D., Lai L.T., Simon J., BorchardtP., Frank R.K., Wu K., Pellegrini V., Curcio M.J., Miederer M., Bander N.H., Sheinberg D.A. ,"Tumor Therapy with Targeted Atomic Nano generators, "Science 294, 16 November 2001, pp. 1537-1540, Nov. 2001.

[35] Kumar M.N.V.R., "Nano and Micro particles as Controlled Drug Delivery Devices", J. Pharmacy Pharmaceutical Science, 3(2):234-258, 2000. 
[36] Adelman L.M., "On Constructing A Molecular Computer", DNA Based Computers II: Dimacs Workshop, Jun. 10-12, 1996 (Dimacs Seriesin Discrete Mathematics and Theoretical Computer Science, V. 44), American Mathematical Society, 1996, pp. 1-21.

[37] Zhang M., Saharawi C.L., Tao W., Tarn T.J., XiN., LiG., "Interactive DNA Sequence and Structure Design for DNA Nano applications", IEEE Transactions on Nano bioscience, Vol. 3, No. 4, Dec.2004.

[38] Chatterjee B., Sachdev M., "Design of a 1.7-GHzLow- Power Delay-Fault-Testable 32-b ALU in 180-nm CMOS Technology", IEEE Transactions on VeryLarge Scale Integration (VLSI) Systems, Vol. 13, no. 11, Nov. 2005.

[39] Hagiya M., "From Molecular Computing to Molecular Programming", in Proc. $6^{\text {th }}$ DIMACS Workshop on DNA Based Computers, Leiden, the Netherlands, and pp. 198-204. June 2004

[40] Sun J., GAO M., Feldman J., "Electric Field Directed Layer-by-Layer Assembly of Highly

[41] Fluorescent CdTe Nanoparticles", J. of Nanoscience and Nanotechnology, 1(2):133-136, Jun. 2001.

[42] Cavalcanti A., Hogg T., Kretly L.C., "Transducers Development for Nanorobotics Applications in Biomedical Engineering", IEEE NDSIConf. On Nanoscale Devices and System Integration, Houston TX, USA, April 2005.

[43] Stracke R., Böhm K.J., Burgold J., Schacht H.,UngerE.,"Physicaland Technical Parameters Determining the Functioning of a Kinesin-Based Cell-Free MotorSystem", Nanotechnology, 11(2):52-56, Jun. 2000.

[44] Reppesgaard L., "Nanobiotechnologie: DieFeinmechaniker der Zukunft nutzen Biomaterial ales biomolecular motors", Biomedical Microdevices, 2:179-184, 2000.

[45] Whitcomb L.L., "Underwater Robotics: Out of theResearch Laboratory and into the Field", pinprick. IEEE Int'l Conf. on Robotics and Automation, a Francisco, CA, USA, pp. 709-716., apr 2002

[46] Cavalcanti A., Hogg T., Shirinzadeh B.,"Nanorobotics System Simulation in 3D Workspaces with Low Reynolds Number",IEEE-RASMHS Int'ISymposium on Micro-Nanomechatronics and Human Science, Nagoya, Japan, Nov. 2006.

[47] Braff D., "Fast contact force computation for no penetrating rigid bodies", in Computer GraphicsProceedings, Annual Conf. Series. ACM SIGGRAPH, pp. 23-34, 1994.

[48] Mitch B., Canny J., "Impulse-based simulation of rigid bodies", Proc. of Symposium on Interactive3DGraphics, pp. 392-398, 1995.

[49] Wall C., Henrich D., and Wörn H., "Parallel on-line Motion Planning for Industrial Robots," 3rdASCESpecialty Conf. on Robotics for Challenging Environments, Robotics 98, pp. 308-314, New Mexico, USA, 1998.

[50] 50) Fukuda T., Arai T., "Prototyping Design and Automation of Micro/Nano Manipulation System," Proc. of IEEE Int'l Conf. on robotics and Automation (ICRA'00), Vol. 1, pp. 192-197, 2000.

[51] Fann J.I., Goar F.J.S., Komtebedde J., Oz M.C., Block P.C., Foster P.C., J. Butany, T. Feldman, T.A.Burdon, "Beating Heart Catheter-Based Edgeto-Edge Mitral Valve Procedure in a Porcine Model: Efficacy andHealing Response", Circulation, 110:988-993, Aug 2004, circ.ahajournals.org/cgi/content/full/110/8/988

[52] Stefanie's C., Toutouzas K., Tsiamis E., StratosC., Vavuranakis M., Kallikazaros I., PanagiotakosD.,Toutouzas P., "Increased Local Temperature in Human Coronary Atherosclerotic Plaques: Anls independent Predictor of Clinical Outcome in Patients Undergoing a Percutaneous Coronary Intervention", J Am Cull Cardiol,37(5):1277-1283, Apr. 2001.

[53] Stefanadis C., Diamantopoulos L., Dernellis J.,Economou E., Tsiamis E., Toutouzas K., VlachopoulosC., Toutouzas P., "Heat Production ofAtherosclerotic Plaques and Inflammation Assessed by the Acute Phase Proteins in Acute Coronary Syndromes",J Mol Cell Cardiol, 32(1):43-52, Jan.2000. 
[54] Ito T., Ikeda U., "Inflammatory cytokines and cardiovascular disease", Current Drug TargetsInflammation and Allergy, 2(3):257-265, Sep. 2003.

[55] Xu C., Wootton D.M., "Platelet Near-Wall Excess in Porcine Whole Blood in Artery-Sized Tubes Under Steady and Pulsatile Flow Conditions", Bioarcheology41 (2):113-125, Apr. 2004.

[56] Cavalcanti, L. Rosen, L. C. Kretly, Moshe. Rosenfeld, Shmuel Einav, "Nanorobotics Challenges in Biomedical Applications, Design and Control", IEEE ICECS Int'l Conf. on Electronics, Circuits and Systems,Tel-Aviv, Israel, December 2004.

[57] Cavalcanti A., "Assembly Automation with Evolutionary Nano robots and Sensor-Based Control applied to Nano medicine", IEEE Transactions on Nanotechnology, Vol. 2, no. 2, pp. 82-87, June 2003

[58] Cavalcanti A. and Freitas R.A. Jr., "Autonomous multi-robot sensor-based cooperation for Nano medicine," Int'l J. "Nonlinear Science Numerical Simulation”, Vol. 3, No.4, pp.743-746, August 2002 ,

[59] 59) Drexler K.E., "Nano systems: molecular machinery, manufacturing, and computation", John Wiley \&Sons, 1992.

[60] Fishbine G., "The Investor's Guide to Nanotechnology \& Micro machines", John Wiley \&Sons, 2001.

[61] Freitas R.A. Jr., "Nano medicine", Vol. I: Basic Capabilities," Lands Bioscience, 1999,http://www.nanomedicine.com.

[62] Geppert L., "The Amazing Vanishing Transistor Act, "Cover story, IEEE Spectrum Magazine, pp. 28-33, October 2002.

[63] Haiyan M., "From Molecular Computing to Molecular Programming," Proc. 6th DIMACS Workshop on DNA Based Computers, pp. 198-204, Leiden, Netherlands, 2000.

[64] Hellman's A., "German Team Creates New Type of Transistor-Like Device," News Analysis, IEEE Spectrum Magazine, pp. 20-21, January 2003.

[65] Kube C.R. and Zhang H., "Task Modelling in Collective Robotics," Autonomous Robots, 4(1), pp.53-72, 1997.

[66] Mokhoff N., "Education Overhaul Urged for Nanotech Revolution", EE Times, Feb. 2003, http://www.theworkcircuit.com/news/OEG20030206S0026.

[67] Moore S.K., "Just One Word - Plastics," Special R\&D Report, Organic Electronics, IEEE Spectrum Magazine, pp.55-59, September 2002.

\footnotetext{
*Corresponding author.

E-mail address: rajumerugu01@ gmail.com
} 